\title{
The Study of Prediction of Sensor System Errors for
}

\section{Maneuvering Target Tracking}

\author{
He Guangjun ${ }^{1, a}$, Li Yanbin ${ }^{1, b}$, Wu Jianfeng ${ }^{1, c}$ and Liu Bin ${ }^{1, *}$ \\ ${ }^{1}$ Air and Missile Defense College, Air Force Engineering University, Xi'an, Shaanxi, Postcode \\ 710051, China \\ aguangjunhe@sina.com, byuanchen4@sina.com, ‘wjf1331@163.com, ‘277306879@qq.com
}

Keywords: maneuvering target tracking; system errors prediction; stochastic jump system Abstract: Considering that it's difficult to build target motion model in structure stochastic jump system, this paper studies system errors estimation. Based on the Maximum Likelihood (ML) estimation, a conclusion is drawn, that is, there exists a causal conjunction between system errors and state estimation. This paper proposes an algorithm to estimate system errors on line by combining an improved estimation algorithm of state parameters based on bootstrap filter and ML estimation. This demonstrates algorithm can be well adapted for non-linear and non-Gaussian problems. The simulation demonstrates the proposed algorithm is feasible and effective to estimate system errors for structure stochastic jump system when statistical property of system errors is considered.

\section{Introduction}

In the process of maneuvering target tracking, sensor measurement errors include system errors and random errors. System errors affect the target position estimation significantly and so does acceleration estimate of target and eventually influence tracking target. In sensor network for fusing estimation of target status, the prediction and tracking system is usually needed to predict target's system errors to improve the estimation performance.

The key topics of predicting system errors in tracking maneuvering target are the loss of target due to the maneuvering and external disturbance and system stochastic switching. This type of switch will make system structure and parameters jump dramatically randomly and make target tracking a challenge. However, with the application of structure stochastic jump system theory, the problem can be solved well.

\section{Parameter Estimation of System Errors}

Considering the system as follows:

$$
\begin{gathered}
\boldsymbol{x}(\mathrm{k}+1)=\boldsymbol{f}[\boldsymbol{x}(k)]+\boldsymbol{w}(k, s) \\
\boldsymbol{z}(k)=\boldsymbol{h}[\boldsymbol{x}(\mathrm{k})]+\mathbf{b}(k, s)+\boldsymbol{v}(\mathrm{k}, \mathrm{s})
\end{gathered}
$$

Where $\boldsymbol{x}(k)$ is system state vector, $\boldsymbol{w}(k, s)$ and $\boldsymbol{v}(k, s)$ are system noise and observation noise respectively, $\boldsymbol{f}(\bullet)$ and $\boldsymbol{h}(\bullet)$ are the known non-linear function, $\boldsymbol{b}(k, s)$ is sensor system errors 
which are either time-variant or time-invariant, $s(k) \in S=\overline{1, \mathrm{M}}$ is M Markov chains that has finite states, and its transition probability is $q[s, k+1 \mid \boldsymbol{x}(k), r, k]=q^{s r}(\boldsymbol{x}, k+1, k)$ 。 Assuming $v(k, s) \sim N(0, R(k, s))$ and $v(k, s) \sim N(0, Q(k, s))$ are independent process noise and measurement noise respectively, $\boldsymbol{Q}(\mathrm{k}, \mathrm{s})$ and $\boldsymbol{R}(k, s)$ are variance of process noise and variance of measurement noise respectively of target motion model s at time $\mathrm{k}$.

Assuming sensor system errors obey normal distribution, and its likelihood function is

$$
q(z(\mathrm{k}) \mid \boldsymbol{b})=\frac{1}{\sqrt{(2 \pi)^{m}|\boldsymbol{R}(\mathrm{k})|}} \exp \left[-\frac{1}{2}(\mathrm{z}(\mathrm{k})-\mathrm{hx}(\mathrm{k})-\mathrm{b})^{\mathrm{T}} \bullet \mathrm{R}^{-1}(\mathrm{k}) \bullet(\mathrm{z}(\mathrm{k})-\mathrm{hx}(\mathrm{k})-\mathrm{b})\right]
$$

If $\boldsymbol{R}(\mathrm{k}, \mathrm{s})=\sigma^{2} \boldsymbol{I}(\mathrm{s})$, and $\sigma$ is a constant, then ( 3 ) will be

$$
q(z(k) \mid b)=\frac{1}{\sqrt{(2 p)^{m}|R(k)|}} \exp \left[-\frac{1}{2 \sigma^{2}} \bullet\|z(k)-h x(k)-b\|^{2}\right]
$$

Where $\|\bullet\|$ means the 2-norm of a matrix. Solving the logarithm of maximum-likelihood function $q(z(\mathrm{k}) \mid \boldsymbol{b}(\mathrm{k}, \mathrm{s}))$, we have:

$$
\lg q(z(\mathrm{k}) \mid \boldsymbol{b})=-\frac{1}{2} \lg \left((2 \pi)^{m}\left|\boldsymbol{\sigma}^{2} \boldsymbol{I}(\mathrm{m}, \mathrm{s})\right|\right)-\frac{1}{2 \sigma^{2}} \bullet\|z(\mathrm{k})-\boldsymbol{h} \boldsymbol{x}(\mathrm{k})-\mathbf{b}\|^{2}
$$

Ignoring the constant, the target maximum-likelihood function value can be expressed as follows:

$$
\max \lg q(z(\mathrm{k}) \mid \boldsymbol{b})=\min _{\boldsymbol{x}, \boldsymbol{b}}\|z(\mathrm{k})-\boldsymbol{h} \boldsymbol{x}(\mathrm{k})-\mathbf{b}\|^{2}
$$

Supposing

$$
g(k)=\|\boldsymbol{z}(\mathrm{k})-\boldsymbol{h} \boldsymbol{x}(\mathrm{k})-\mathbf{b}\|^{2}
$$

Getting the maximum value of maximum-likelihood function is equivalent to getting the minimum value of $g(k)$. Now by making partial derivative of $g(k)$ with respect to $\boldsymbol{x}(\mathrm{k})$ equals zero, we can get the estimation value $\hat{\boldsymbol{x}}(\mathrm{k})$ of $\boldsymbol{x}(\mathrm{k})$

$$
\begin{gathered}
\frac{\partial g(k)}{\partial \boldsymbol{x}(k)}=-2 \boldsymbol{h}^{T}(z(\mathrm{k})-\boldsymbol{h} \boldsymbol{x}(\mathrm{k})-\mathbf{b})=0 \\
\hat{\boldsymbol{x}}(k)=\left(\boldsymbol{h}^{T} \boldsymbol{h}\right)^{-1} \boldsymbol{h}^{T}(z(\mathrm{k})-\mathbf{b})
\end{gathered}
$$

Substituting (8) into (1) and minimizing $g(k), \hat{\mathbf{b}}(k, s)$ can be estimated as:

$$
\hat{\mathbf{b}}(k, s)=\boldsymbol{z}(\mathrm{k})-\boldsymbol{h} \hat{\boldsymbol{x}}(k)
$$


So system errors $\hat{\mathbf{b}}(k, s)$ can be derived by solving $\hat{\boldsymbol{x}}(\mathrm{k})$ under the state of $s$ in formula (9).

\section{State Parameter Estimation Algorithm Based on Improved Bootstrap Filter}

EKF(Extended Kalman filter) is a basic linear filter in non-linear system. However, it can be not applied well in real-time circumstance due to information loss in the process of linearizing. Gordon and Doucet, et al proposed (BSF) ${ }^{[5,6]}$. The main idea of BSF is expressing state probability density function by random sample sets. Practice indicates BSF is much more accurate than EKF and if the sampling number $N$ is suitable, it operates faster than EKF too, so it can be applied to non-linear system ${ }^{[4]}$. Now for stochastic jump system with system errors in (1)(2), we propose a filter algorithm to estimate $\hat{\boldsymbol{x}}_{k}$. The new algorithm can be described as follows:

( 1 ) According to the known probability density function $q[x(0)]$ of system state vector, we sample randomly to get random sample set $\quad\left\{\boldsymbol{x}_{i}(0), i=\overline{1, N}\right\}$, $q[\boldsymbol{x}(0)]=\sum_{i=1}^{M} q[\boldsymbol{x}(0) \mid s(0)=i] q[s(0)=i]$ and its initial condition is $q[\boldsymbol{x}(0) \mid s(0)], q[s(0)]$. Estimate $\hat{\boldsymbol{b}}_{i}(0)$ based on actual situation.

( 2 ) According to the known probability density function $q[x(0)]$ of systematic noise state vector, we sample randomly to get the random sample set $\left\{\boldsymbol{w}_{i}^{(s)}(k), i=\overline{1, N}, s=\overline{1, M}\right\}$.

( 3 ) According to state equation, we get the one-step predicting sample $\overline{\boldsymbol{x}}_{i}^{(s)}(k+1)=\boldsymbol{f}\left[\boldsymbol{x}_{i}(k), s(k), \boldsymbol{w}_{i}^{(s)}(k)\right]$

( 4 ) Getting the sample $\boldsymbol{x}_{i}^{*}(k+1)=\sum_{j=1}^{M} \overline{\boldsymbol{x}}_{i}^{s}(k+1) f\left[s(k)=j \mid \boldsymbol{Z}^{k}\right]$.

( 5 ) Calculating the normalized weighting factor using Smith sampling probability factor formula, that is, probability factor $q_{i}(k+1)$ and probability density function $q_{i}(k+1)$.

$$
q_{i}(k+1)=f\left[\boldsymbol{x}_{i}^{*}(k+1) \mid \boldsymbol{Z}^{k+1}\right]=\frac{\sum_{j=1}^{M} \boldsymbol{A}^{*}\left[\boldsymbol{x}_{i}^{*}(k+1), s(k+1)=j, \boldsymbol{z}_{k+1}\right]}{\sum_{j=1}^{M} \sum_{i=1}^{N} \boldsymbol{A}^{*}\left[\boldsymbol{x}_{i}^{*}(k+1), s(k+1)=j, \boldsymbol{z}_{k+1}\right]}
$$

( 6 ) Extracting random samples $\{u(i), i=\overline{1, N}\}$ from uniform distribution $(0,1)$, and resampling by $q_{i}(k+1)$ to obtain the new random sample $\boldsymbol{x}_{i}(k+1)=\boldsymbol{x}_{l}^{*}(k+1)$. 
( 7 ) Calculating system state and structure state estimation values and the corresponding variance as follows:

$$
\begin{gathered}
\boldsymbol{x}(k+1 \mid k+1)=\frac{1}{N} \sum_{i=1}^{N} \boldsymbol{x}_{i}(k+1) \\
\hat{s}(k+1)=\arg \max _{s \in S} f\left[s(k+1) \mid \boldsymbol{Z}^{k+1}\right] \\
\boldsymbol{P}(k+1 \mid k+1)=\frac{1}{N} \sum_{i=1}^{N}\left[\boldsymbol{x}_{i}(k+1)-\boldsymbol{x}(k+1 \mid k+1)\right]\left[\boldsymbol{x}_{i}(k+1)-\boldsymbol{x}(k+1 \mid k+1)\right]^{T}
\end{gathered}
$$

The bootstrap filter can be realized by executing (2) (7) recurrently.

We give an initial value to $\hat{\boldsymbol{b}}_{k, s}$ and it can be regard as a constant implicitly in iterative formula above. If the covariance value is larger than a threshold value in iterative calculation, it should be reset.

\section{Simulation and Analysis}

To verify the proposed algorithm, assuming sensor measurement errors change dramatically due to external interference (for example, radio interference), that is, noise is included in observation equation:

$$
z_{1}(k+1)=h_{1}(x(k+1))+v_{1}(s, k+1) \quad(s \in S=1,2)
$$

where $v_{1}(s, k+1)$ is white noise vector, $v_{1}(2, k+1)$ is interference noise, measurement noise $v_{2} \sim N\left(0, R_{2}(k)\right)$. The initial conditions of systematic noise, observation noise and system state are

$$
\begin{gathered}
\boldsymbol{w}(k) \sim \boldsymbol{N}[\boldsymbol{w}(k) \mid 0, \boldsymbol{Q}], \boldsymbol{x}_{0}^{(s)} \sim \boldsymbol{N}\left[\boldsymbol{x}_{0}^{(s)} \mid \boldsymbol{m}_{0}^{(s)}, \Theta_{0}^{(s)}\right], \boldsymbol{v}_{1}(k, 1) \sim \boldsymbol{N}\left[\boldsymbol{v}_{1}(k, 1) \mid \mathbf{0}, \boldsymbol{R}_{1}(k, 1)\right] \\
\boldsymbol{v}_{1}(k, 2) \sim \boldsymbol{N}\left[\boldsymbol{v}_{1}(k, 2) \mid \mathbf{0}, \boldsymbol{R}_{1}(k, 2)\right], \boldsymbol{R}_{1}(k, 1)<<\boldsymbol{R}_{1}(k, 2)
\end{gathered}
$$

The transition probability of structure state is:

$$
q^{(s r)}(k+1, k)=q(s, k+1 \mid r, k)=q(s) \quad(s=1,2)
$$

$\boldsymbol{w}(k)$ is zero-mean Gaussian random sequence with variance $\boldsymbol{Q} \cdot w_{1}, w_{2}$ are independent with the same variance $\sigma_{\alpha}^{2}$, so $\boldsymbol{Q}=\boldsymbol{\sigma}_{\alpha}^{2} \boldsymbol{I}, E[\boldsymbol{w}(k)]=\mathbf{0}, E\left[\boldsymbol{w}(k) \boldsymbol{w}^{\mathrm{T}}(j)\right]=\boldsymbol{Q} \boldsymbol{\delta}_{k j}$. Supposing $\boldsymbol{R}_{1}(1, k)=\operatorname{diag}\left[0.36 \times 10^{4}(\mathrm{~m})^{2}, 3 \times 10^{-6}(\mathrm{rad})^{2}\right], T=20 \mathrm{~ms}, \lambda=0.05$, $m_{0}^{s}=\left[\begin{array}{lllll}10000 \mathrm{~m} & 300 \mathrm{~m} / \mathrm{s} \quad 4000 \mathrm{~m} \quad 150 \mathrm{~m} / \mathrm{s} \quad 5 \mathrm{~m} / \mathrm{s}^{2} \quad 4 \mathrm{~m} / \mathrm{s}^{2}\end{array}\right]^{T}, \quad \sigma_{\alpha}^{2}=100, R_{2}(k)=1 \times 10^{-6}(\mathrm{rad})^{2}$, $\boldsymbol{R}_{1}(2, k)=\operatorname{diag}\left[9.0 \times 10^{4}(\mathrm{~m})^{2}, 7.5 \times 10^{-6}(\mathrm{rad})^{2}\right]$,

the simulation results are shown in figure 1 to 3 by applying the advanced bootstrap filter based on stochastic jump system. 


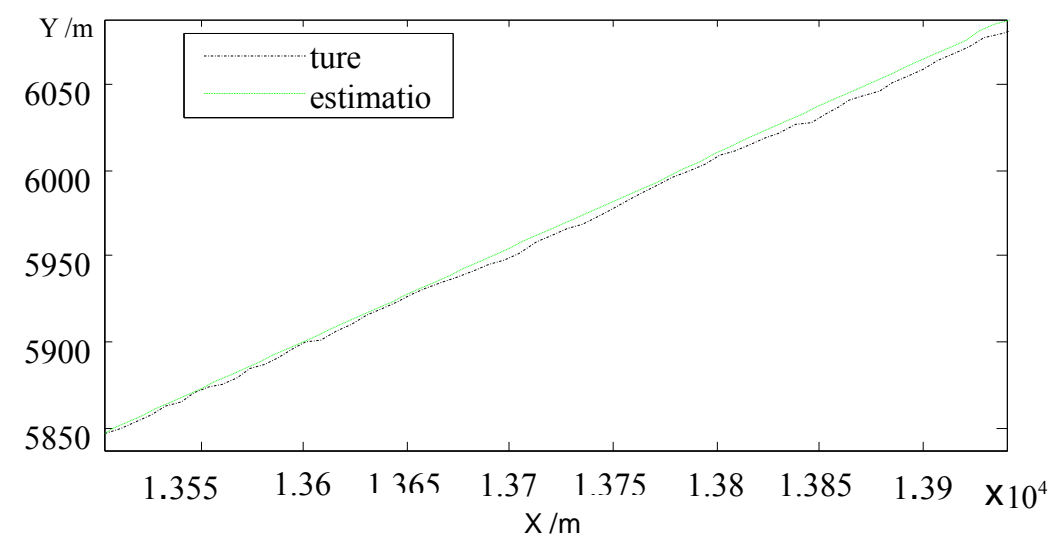

Fig.1 the true and estimated target trajectories with stochastic jump system errors

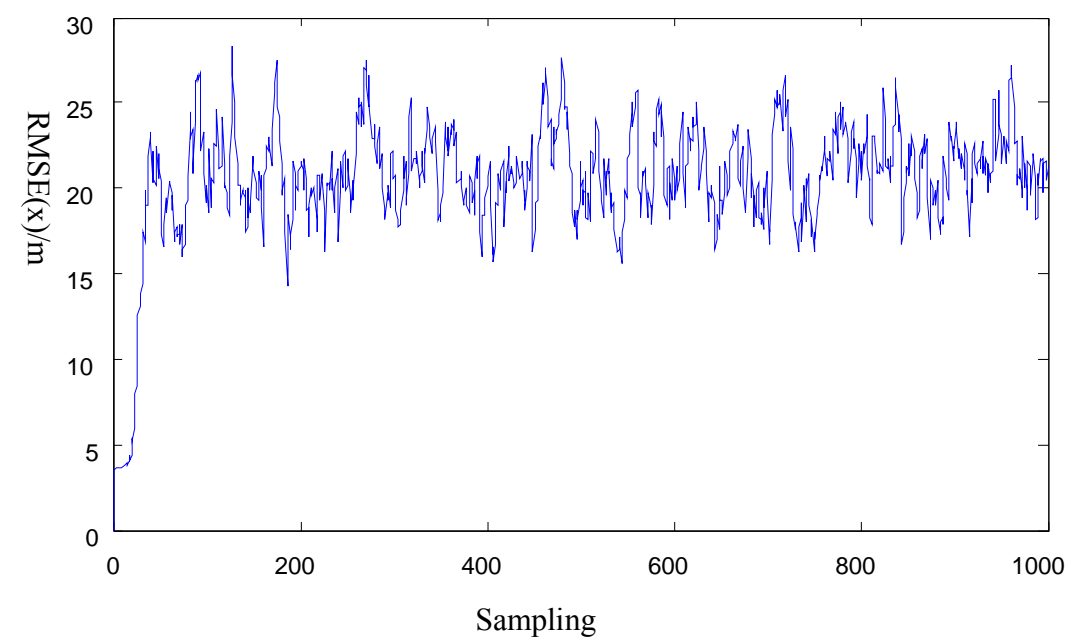

Fig.2 RMSE of estimated systematic error in X- direction with stochastic jump system errors

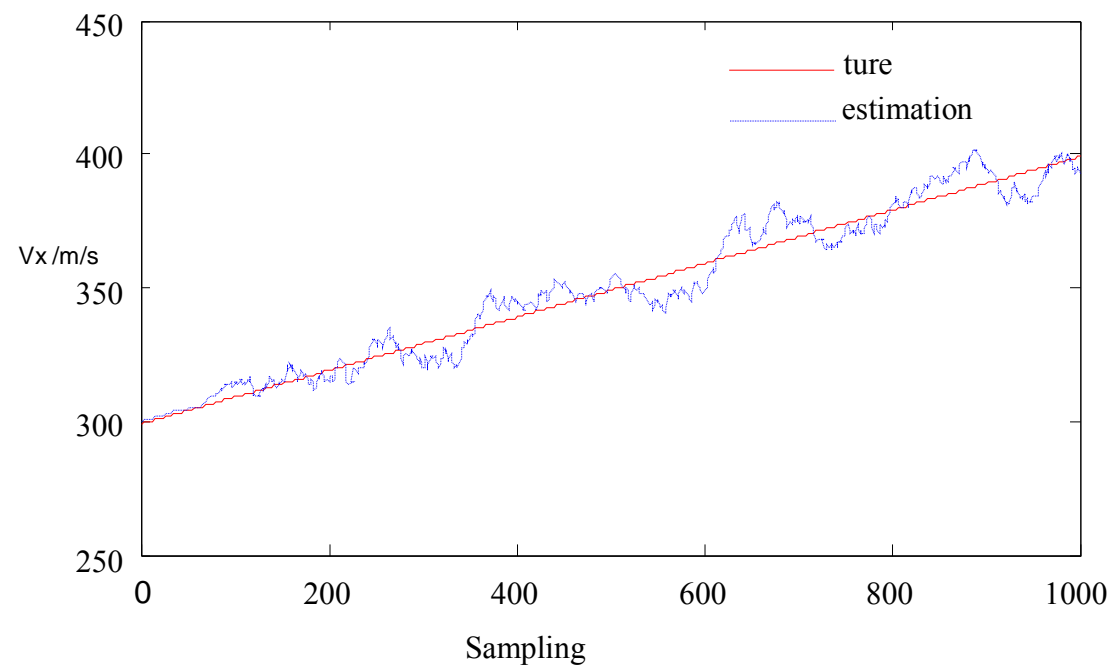

Fig.3 RMSE of estimated velocity error in X- direction with stochastic jump system errors

It can be concluded from the figures that target's estimation of position, speed and acceleration are close to the real value well. From figure1, RMSE of system errors for estimating position in $\mathrm{X}$ direction location with stochastic jump system is nearly approximately 20 meters. For each 
sampling time, as the state switchs, system errors of state's each node will converge to a point and this point will be used as the input to switch node of the next state.

\section{Conclusions}

Considering that it's difficult to build target motion model of target in structure stochastic jump system, this paper studies system errors estimation. We propose an estimation algorithm on line by combining status parameters estimation based on advanced bootstrap filter and Maximum Likelihood (ML) estimation. Based on ML, we propose there exists a causal conjunction between system errors and state estimation. An advanced bootstrap filter based on random sampling is applied to stochastic jump system. This novel algorithm can be well adapted to non-linear and non-Gaussian problems and overcomes the key topics involved by the traditional filters. The algorithm can improve the overall performance of filter. Simulation for time-variant and time-invariant system verifies is feasibility and validity.

\section{REFERENCES}

[1] WU Sentang ZHANG Shuixiang CHEN Haier. Approach for Adaptive Filter of Systems with Random Changing Structures. Journal of Beijing University of Aeronautics and Astronautics. Vol.28 No.3 June 2002: 287-289.

[2] Bar-Shalom Y, Li X R. Estimation and tracking principles techniques and software. Boston: Artech House, 1993.

[3] Pan Fuchen, Li Lin. A Non-linear Bayesian Dynamic Model Algorithm Studying. ,China Science and Technology Information. No.22.Nov. 2010:99-100.

[4] El-Kebir B,Ahmad H.Exponential stability of singular systems with multiple time-varying delays[J].Automatic,2009,45(2):539-545..

[5] Wang Xianghua, Tan Zheng, Yang Huijie and Yang Xinyu, A fuzzy adaptive algorithm based on current statistical model for maneuvering target tracking, Acta Armamentarii, vol.30, No.8, pp.1089-1093, 2009.

[6] CHEN Jia, FANG Yang-wang, LOU Shun-tian. Investigation about $\mathrm{H}_{\infty}$ stability of linear singular perturbed system with Markovian jumping structure [J]. Control and Decision, 2013, 28 (2): 279-284. 